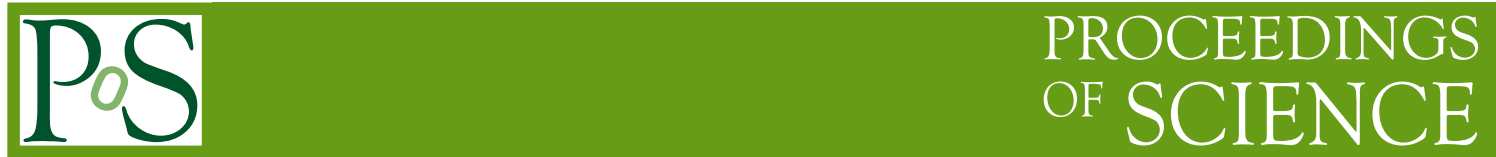

\title{
Quarkonium production at the Tevatron and the LHC
}

\author{
Pierre Artoisenet* \\ The Ohio State University \\ E-mail: partois@pacific.mps.ohio-state.edu
}

NRQCD predictions for the production rates of $J / \psi$ and $\psi(2 S)$ at the LHC are presented. We consider a data-driven approach to reduce the theoretical uncertainties. The predicted cross sections differential in transverse momentum offer the opportunity to further test the color-octet dominance in $J / \psi$ or $\psi(2 S)$ hadroproduction.

35th International Conference of High Energy Physics - ICHEP2010,

July 22-28, 2010

Paris France

${ }^{*}$ Speaker. 


\section{Introduction}

Since the discovery of the $J / \psi$, quarkonium states has attracted a vivacious interest. In particular, the production of these states offers a challenging opportunity to test our understanding of QCD. The production of quarkonia can be studied in the framework of NonRelativitic QCD [1], an effective field theory that separates the perturbative and non-perturbative energy scales. Recently, most of the short-distance coefficients appearing in the NRQCD expansion of cross sections for quarkonium hadroproduction have been extended at NLO accuracy in $\alpha_{s}[2,3,4,5,6,7,8]$. Despite these recent theoretical advances, we are still lacking a clear picture of the mechanisms at work in quarkonium hadroproduction. As several quarkonium production rates are currently being measured at the LHC, it is useful to compare these early data to some benchmark predictions. In this analysis, we restrict ourself to the direct production of $J / \psi$ and $\psi(2 S)$, and use a scheme that is relevant to test the color-octet ${ }^{3} S_{1}$ dominance at large $p_{T}$ against the measured production rate differential in $p_{T}$.

\section{Framework}

The differential cross sections are computed at order $\alpha_{s}^{3}$ (leading order). The relevant partonlevel processes are $g g \rightarrow g c \bar{c}[n], g q \rightarrow q c \bar{c}[n], g \bar{q} \rightarrow \bar{q} c \bar{c}[n]$ and $q \bar{q} \rightarrow g c \bar{c}[n]$. The color-singlet channel $n={ }^{3} S_{1}^{[1]}$ and the color-octet channels $n={ }^{3} S_{1}^{[8]},{ }^{1} S_{0}^{[8]}$ are taken into account ${ }^{1}$. The curves are generated with MadOnia [9]. We consider the PDF set cteq611 and the running of $\alpha_{s}$ at one loop accuracy, with $\alpha_{s}\left(M_{Z}\right)=0.13$.

In the case of the color-octet ${ }^{3} S_{1}$ channel, the logarithms $\alpha_{s} \log \left(m_{c} / p_{T}\right)$ are resummed by calculating the cross section $d \sigma^{f r} / d p_{T}$ in the fragmentation approximation and by solving the DGLAP evolution equation for the fragmentation funtion $D_{g \rightarrow c \bar{c}_{8}\left({ }^{3} S_{1}\right)}\left(\mu_{f r}\right)$. For this calculation, we use the code from [11]. In the evolved cross section, the fragmentation scale $\mu_{f r}$ is set equal to the renormalization and the factorization scales. Following the approach in [12], the rescaling factor

$$
R=\frac{d \sigma^{f r} / d p_{T}\left(\mu_{f r}=\mu_{r}\right)}{d \sigma^{f r} / d p_{T}\left(\mu_{f r}=2 m_{c}\right)}
$$

is applied to the spectrum $d \sigma\left({ }^{3} S_{1}^{[8]}\right) / d p_{T}$ calculated at fixed order in $\alpha_{s}$.

The NRQCD Long-Distance Matrix Elements (LDME) $\left\langle\mathscr{O}_{8}\left({ }^{3} S_{1}\right)\right\rangle$ and $\left\langle\mathscr{O}_{8}\left({ }^{1} S_{0}\right)\right\rangle$ are extracted from the Tevatron data as described in the next section. For the mass of the charm quark, we use the central value $m_{c}=1.5 \mathrm{GeV}$ and allow for variation of $0.1 \mathrm{GeV}$. The renormalization and factorization scales are kept equal. We use the central value $\mu_{r}=\mu_{f}=\mu_{0}=\sqrt{4 m_{c}^{2}+p_{T}^{2}}$ and allow for variation of a factor 2 .

\section{Extraction of the color-octet LDME's at the Tevatron}

The cross section differential in $p_{T}$ for the production of direct $J / \psi$ at the Tevatron (run I) has been measured by the CDF collaboration [13]. The energy of the collisions is $\sqrt{s}=1.8 \mathrm{TeV}$,

\footnotetext{
${ }^{1}$ At leading order in $\alpha_{S}$, the P-wave color-octet channels $n={ }^{3} P_{J}^{[8]}$ can be effectively described by shifting the Long-Distance Matrix Element associated with the ${ }^{1} S_{0}^{[8]}$ channel.
} 

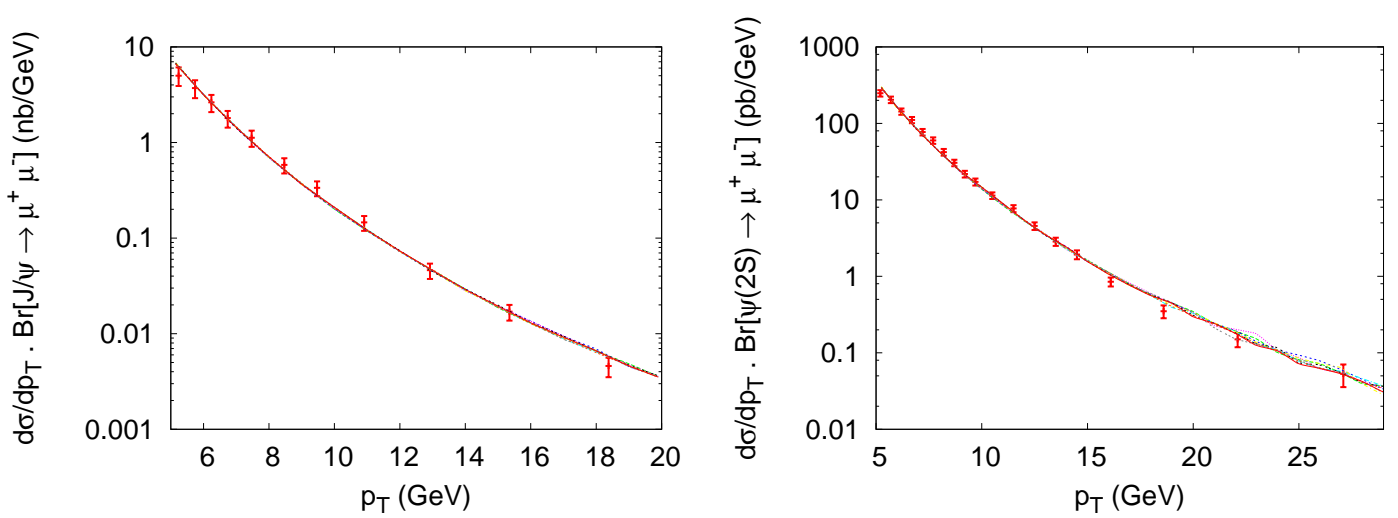

Figure 1: Left: direct production of $J / \psi$ at the Tevatron, run I. The errorbars are the data collected by the CDF collaboration. The curves are the NRQCD predictions, with the values of the color-octet LDME's fitted to the central values of the CDF data points. Right: same plot for prompt $\psi(2 S)$ production at the Tevatron, run II.

\begin{tabular}{c|ccc||ccc} 
& \multicolumn{3}{c}{$\left\langle\mathscr{O}_{8}^{J / \psi}\left({ }^{3} S_{1}\right)\right\rangle\left(10^{-3} \times \mathrm{GeV}^{3}\right)$} & \multicolumn{3}{c}{$\left\langle\mathscr{O}_{8}^{J / \psi}\left({ }^{1} S_{0}\right)\right\rangle\left(10^{-2} \times \mathrm{GeV}^{3}\right)$} \\
scale $/ m_{c}$ & $1.4 \mathrm{GeV}$ & $1.5 \mathrm{GeV}$ & $1.6 \mathrm{GeV}$ & $1.4 \mathrm{GeV}$ & $1.5 \mathrm{GeV}$ & $1.6 \mathrm{GeV}$ \\
\hline $0.5 \mu_{0}$ & 2.45 & 2.72 & 2.95 & 3.29 & 4.12 & 5.05 \\
$\mu_{0}$ & 5.56 & 6.12 & 6.71 & 4.14 & 5.41 & 6.77 \\
$2 \mu_{0}$ & 11.0 & 12.5 & 13.9 & 5.59 & 7.30 & 9.08 \\
\hline \hline$\mu_{0}$, upper edge & \multicolumn{3}{c}{7.12} & & 6.57 & \\
$\mu_{0}$, lower edge & & 4.94 & & & 4.26 &
\end{tabular}

Table 1: Upper: values of the color-octet LDME's for direct $J / \psi$ production extracted from the fit to the central values of the CDF data points. Lower: values of the color-octet LDME's extracted from the fit to the upper and lower edge of the errorbars reported by the CDF collaboration, after setting the mass of the charm quark and the scales to their central values: $m_{c}=1.5 \mathrm{GeV}, \mu=\mu_{0}$.

and the kinematic region is defined by the cut on pseudo-rapidity $|\eta(J / \psi)|<0.6$. The measured spectrum is shown in Figure 1 (left).

Considering these kinematic conditions, the short-distance coefficients $d \hat{\sigma}_{1}\left({ }^{3} S_{1}\right) / d p_{T}, d \hat{\sigma}_{8}\left({ }^{3} S_{1}\right) / d p_{T}$, $d \hat{\sigma}_{8}\left({ }^{1} S_{0}\right) / d p_{T}$ as well as the rescaling factor $R$ are calculated for $m_{c}=1.4,1.5$ and $1.6 \mathrm{GeV}$ and $\mu_{f}=\mu_{r}=\mu_{0} / 2, \mu_{0}$ and $2 \mu_{0}$. A binning of $1 \mathrm{GeV}$ is used to generate each curve through a MonteCarlo integration. To get a continous expression for each curve, the quantities $\log \left[d \hat{\sigma} / d p_{T}\right]$ were extrapolated linearly between the central values of each pair of adjacent bins.

We set $\left\langle\mathscr{O}_{1}^{J / \psi}\left({ }^{3} S_{1}\right)\right\rangle=1.32 \mathrm{GeV}^{3}$ which corresponds to the value of the Long-Distance Matrix Element for $J / \psi$ decay calculated in [10], up to a convention factor $2 J+1=3$. For each of the 9 sets $\left(m_{c}, \mu\right)$ of theoretical inputs, the values of the color-octet LDME's are extracted by fitting the theoretical spectrum to the central values of the CDF data points. The fit is illustrated in Figure 1 (left). The values of the extracted LDME's for each set of theoretical inputs are given in Table 1.

In order to take into account the experimental errors in the fit, the values of the color-octet LDME's are also extracted by fitting the theoretical spectrum to the lower/upper edge of the CDF data points. In that case, the theoretical input parameters are fixed to $m_{c}=1.5 \mathrm{GeV}, \mu_{f}=\mu_{f}=\mu_{0}$. The corresponding values of the color-octet LDME's are given in the last two lines of Table 1. 


\begin{tabular}{c|ccc||ccc}
\multicolumn{1}{c}{} & \multicolumn{2}{c}{$\left\langle\mathscr{O}_{8}^{\Psi(2 S)}\left({ }^{3} S_{1}\right)\right\rangle\left(10^{-3} \times \mathrm{GeV}^{3}\right)$} & \multicolumn{3}{c}{$\left\langle\mathscr{O}_{8}^{\psi(2 S)}\left({ }^{1} S_{0}\right)\right\rangle\left(10^{-3} \times \mathrm{GeV}^{3}\right)$} \\
scale $/ m_{c}$ & $1.4 \mathrm{GeV}$ & $1.5 \mathrm{GeV}$ & $1.6 \mathrm{GeV}$ & $1.4 \mathrm{GeV}$ & $1.5 \mathrm{GeV}$ & $1.6 \mathrm{GeV}$ \\
\hline $0.5 \mu_{0}$ & 1.93 & 2.26 & 2.62 & 4.72 & 7.04 & 9.36 \\
$\mu_{0}$ & 4.07 & 4.79 & 5.37 & 3.41 & 6.71 & 10.5 \\
$2 \mu_{0}$ & 7.70 & 9.04 & 10.4 & 3.73 & 7.99 & 12.0 \\
\hline \hline$\mu_{0}$, upper edge & \multicolumn{3}{c}{5.46} & & 6.48 & \\
$\mu_{0}$, lower edge & & 4.12 & & & 6.95
\end{tabular}

Table 2: Upper: values of the color-octet LDME's for $\psi(2 S)$ production extracted from the fit to the central values of the CDF data points. Lower: values of the color-octet LDME's extracted from the fit to the upper and lower edge of the errorbars reported by the CDF collaboration, after setting the mass of the charm quark and the scales to their central values: $m_{c}=1.5 \mathrm{GeV}, \mu=\mu_{0}$.

We apply the same procedure to extract the color-octet LDME's for $\psi(2 S)$ production, using the CDF data [14] collected at the Tevatron, run II $(\sqrt{s}=1.96 \mathrm{TeV},|y[\psi(2 S)]|<0.6)$, and setting $\left\langle\mathscr{O}_{1}^{\psi(2 S)}\left({ }^{3} S_{1}\right)\right\rangle=0.758 \mathrm{GeV}^{3}$. The fit is illustrated in Figure 1 (right), and the values of the coloroctet LDME's are given in Table 2.

\section{Predicted rates at the $\mathrm{LHC}$}

We now consider the direct $J / \psi$ production rate in proton-proton collisions at the LHC, $\sqrt{s}=7$ $\mathrm{TeV}$. For each set $\left(m_{c}, \mu\right)$ of theoretical inputs, we consider the associated color-octet LDME's given in Table 1 and compute the differential cross section at the LHC. We consider both the rapidity region $|y|<2.4$ and the pseudo-rapidity region $2<|\eta|<5$. The rescaling factor $R$ is calculated within the same acceptance cuts.

The envelope of the curves corresponding to different theoretical inputs is relatively narrow, because the effects from varying $m_{c}$ and $\mu$ are partly cancelled by the variation of the color-octet LDME's. We define the error $d y^{t h,+}$ (resp. $d y^{t h,-}$ ) as the gap between the central curve - associated with $m_{c}=1.5 \mathrm{GeV}, \mu=\mu_{0}$ - and the upper bound (resp. lower bound) of the envelope of the 9 curves corresponding to different theoretical inputs for the scales and the mass of the charm quark.

For the central set $\left(m_{c}=1.5, \mu=\mu_{0}\right)$, we also consider the differential cross sections after setting the color-octet LDME's to the values given in the last two lines of Table 1. The resulting curves are used to estimate the errors $d y^{\exp ,+}$ and $d y^{\text {exp,- }}$ induced by the experimental errors on the data points of Figure 1. We then combine in quadrature the errors $d y^{\text {th }}$ and $d y^{\exp }$. The resulting errors $d y^{+}$and $d y^{-}$are used to define the uncertainty bands shown in Figure 2 (left).

We consider the same approach to predict the $\psi(2 S)$ prompt production rate at the LHC. The resulting uncertainty bands are shown in Figure 2 (right).

\section{Acknowledgment}

This work has been initiated at a workshop on Quarkonium production at CERN in February 2010, and has been shaped by many discussions with Geoffrey Bodwin, Eric Braaten, Fabio Maltoni and Michelangelo Mangano. I especially thank Fabio Maltoni and Geoffrey Bodwin for their help with the code [11] for the DGLAP evolution of the fragmentation process $g \rightarrow c \bar{c}_{8}\left({ }^{3} S_{1}\right)$. 

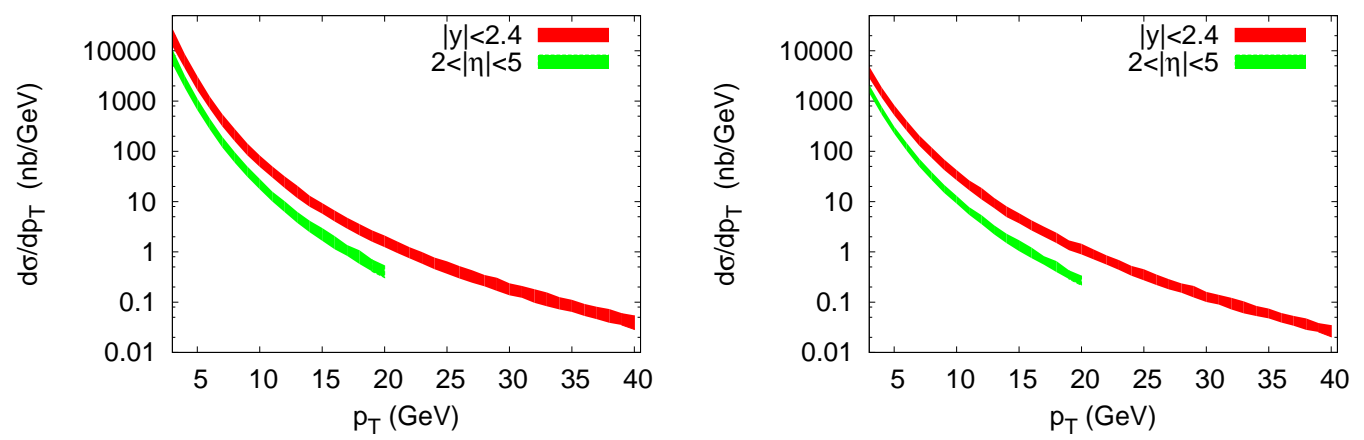

Figure 2: Predicted uncertainty bands for the direct production of $J / \psi$ (left), and for the prompt production of $\psi(2 S)$ (right) at the LHC, $\sqrt{s}=7 \mathrm{TeV}$.

\section{References}

[1] G. T. Bodwin, E. Braaten and G. P. Lepage, Phys. Rev. D 51, 1125 (1995) [Erratum-ibid. D 55, 5853 (1997)] [arXiv:hep-ph/9407339].

[2] J. M. Campbell, F. Maltoni and F. Tramontano, Phys. Rev. Lett. 98, 252002 (2007) [arXiv:hep-ph/0703113].

[3] P. Artoisenet, J. M. Campbell, J. P. Lansberg, F. Maltoni and F. Tramontano, Phys. Rev. Lett. 101, 152001 (2008) [arXiv:0806.3282 [hep-ph]].

[4] B. Gong and J. X. Wang, Phys. Rev. Lett. 100, 232001 (2008) [arXiv:0802.3727 [hep-ph]].

[5] B. Gong, X. Q. Li and J. X. Wang, Phys. Lett. B 673, 197 (2009) [Erratum-ibid. 693, 612 (2010)] [arXiv:0805.4751 [hep-ph]].

[6] Y. Q. Ma, K. Wang and K. T. Chao, arXiv:1002.3987 [hep-ph].

[7] Y. Q. Ma, K. Wang and K. T. Chao, arXiv:1009.3655 [hep-ph].

[8] M. Butenschoen and B. A. Kniehl, arXiv:1009.5662 [hep-ph].

[9] P. Artoisenet, F. Maltoni and T. Stelzer, JHEP 0802, 102 (2008) [arXiv:0712.2770 [hep-ph]].

[10] G. T. Bodwin, H. S. Chung, D. Kang, J. Lee and C. Yu, Phys. Rev. D 77, 094017 (2008) [arXiv:0710.0994 [hep-ph]].

[11] F. Maltoni, A. Petrelli; G. Bodwin, J. Lee

[12] E. Braaten, B. A. Kniehl and J. Lee, Phys. Rev. D 62, 094005 (2000) [arXiv:hep-ph/9911436].

[13] F. Abe et al. [CDF Collaboration], Phys. Rev. Lett. 79, 578 (1997).

[14] T. Aaltonen et al. [CDF Collaboration], Phys. Rev. D 80, 031103 (2009) [arXiv:0905.1982 [hep-ex]]. 